\title{
Massless flows between minimal $W$ models
}

\author{
Clare Dunning] \\ Dept. of Mathematics, University of York, York YO1 5DD, UK
}

\begin{abstract}
We study the renormalisation group flows between minimal $W$ models by means of a new set of nonlinear integral equations which provide access to the effective central charge of both unitary and nonunitary models. We show that the scaling function associated to the nonunitary models is a nonmonotonic function of the system size.
\end{abstract}

1. A recent study of the renormalisation group flows between nonunitary minimal models revealed an unexpected behaviour for the groundstate energy $E_{0}(R)$, in that it was a nonmonotonic function of the system size $R$ [1]. The nonmonotonicity was illustrated using the finite-size scaling function $c_{\mathrm{eff}}(r)$, which up to the bulk term is proportional to the groundstate energy

$$
E_{0}(R)=E_{\text {bulk }}(M, R)-\frac{\pi c_{\mathrm{eff}}(r)}{6 R} \quad, \quad r=M R,
$$

where $M$ is the so-called crossover scale (the mass in massive theories). As the system size goes to zero $c_{\text {eff }}(r)$ becomes the effective central charge

$$
\lim _{r \rightarrow 0} c_{\text {eff }}(r)=c-24 \Delta_{0} .
$$

We denote the actual central charge by $c$ while $\Delta_{0}$ is the conformal dimension of the lowest primary field of the UV CFT.

The effective central charge and the central charge of the unitary minimal models coincide, and according to Zamolodchikov's $c$-theorem [2] there exists a function $\widetilde{c}$ which is monotonic. However, apart from the UV and IR points at which $\widetilde{c}$ equals the central charge of the relevant CFT, it is not clear if there is any connection with $E_{0}(r)$. Nevertheless the groundstate energy of the unitary models is always monotonic. Analogously it had been thought that the groundstate energy of the nonunitary models would also be monotonic, but the results of [1] and [3 6] provide a number of counter examples. In this letter we study a further set of perturbed conformal field theories, demonstrating that $c_{\text {eff }}(r)$ behaves nonmonotonically for the majority of nonunitary models.

We consider the minimal models $W \mathcal{G}_{N}^{p, q}$ based on one of the simply laced Lie algebras $\mathcal{G}=A_{n-1}, D_{n}, E_{6}, E_{7}, E_{8}$ []]. The models are specified by two coprime integers $p$ and $q$ with $p>h$, in terms of which the central charge and the effective central charge are

$$
c=N\left(1-\frac{h(h+1)(p-q)^{2}}{p q}\right) \quad, \quad c_{\mathrm{eff}}=N\left(1-\frac{h(h+1)}{p q}\right) .
$$

Here $N$ denotes the rank of the algebra and $h$ the dual Coxeter number. The primary fields $\Phi_{\Omega, \Omega^{\prime}}$ are labelled by a pair of weights $\Omega, \Omega^{\prime}$ which satisfy

$$
\theta \cdot \Omega \leq q \quad, \quad \theta \cdot \Omega^{\prime} \leq p,
$$

\footnotetext{
${ }^{1}$ tcd1@york.ac.uk
} 
where $\theta$ is the highest root of $\mathcal{G}[\mathbb{8}]$. All models have a primary field $\Phi_{\text {adj }}$ that has weights $\Omega^{\prime}=$ $\Omega_{\text {id }}$ and $\Omega=\Omega_{\text {adj }}$ corresponding to the trivial and adjoint representations of $\mathcal{G}$ respectively. It has conformal dimensions

$$
\Delta=\bar{\Delta}=1-\frac{(q-p) h}{q}
$$

and is relevant for all $p, q$ such that $q>p$. Formally denoting the action of the unperturbed $\mathrm{CFT} \mathcal{A}_{\mathrm{CFT}}$, that of the perturbed model may be written

$$
\mathcal{A}=\mathcal{A}_{\mathrm{CFT}}+\lambda \int d^{2} x \Phi_{\mathrm{adj}}
$$

reproducing for $A_{1}$ the well-known $\phi_{13}$ perturbations of the Virasoro minimal models.

Depending on the sign of the coupling constant $\lambda$, the perturbation either leads to a massive quantum field theory, or it induces a 'massless flow' into a conformal field theory with smaller effective central charge. One of the standard methods of studying the groundstate energy of both types of model is the thermodynamic Bethe ansatz. The result is a set of coupled nonlinear integral equations (the TBA equations), whose solution provides direct access to $c_{\text {eff }}(r)$ at all values of $r$.

The unitary $W \mathcal{G}_{N}^{p, p+1}$ can alternatively be described as $\mathcal{G}_{N}^{k} \times \mathcal{G}_{N}^{1} / \mathcal{G}_{N}^{k+1}$ coset models at $k=p-h$, in terms of which the perturbing operator $\Phi_{\text {adj }}$ is usually known as $\Phi_{\mathrm{id}, \mathrm{id}, \text { adj }}$. TBA equations describing the evolution of the effective central charge between these coset models, and therefore the unitary $W \mathcal{G}$ models, are already known [9 11], and they verify the conjectured pattern of flows [8, 12]:

$$
W \mathcal{G}_{N}^{p, p+1}+\Phi_{\text {adj }} \rightarrow W \mathcal{G}_{N}^{p-1, p}
$$

By dropping the restriction $q=p+1$, we may also consider flows originating from the much larger class of nonunitary $W \mathcal{G}$ minimal models, which also have a description as a coset but at fractional level $\mathcal{G}_{N}^{p /(q-p)-h} \times \mathcal{G}_{N}^{1} / \mathcal{G}_{N}^{p /(q-p)-h+1}$. Analogous to the known behaviour of the $\phi_{13}$ perturbations of the Virasoro models 13 16, it is natural to suppose that the nonunitary generalisation of (7) will be

$$
W \mathcal{G}_{N}^{p, q}+\Phi_{\text {adj }} \rightarrow W \mathcal{G}_{N}^{2 p-q, p}
$$

TBA equations describing massless flows from these nonunitary models are not yet known. Instead, motivated by [1, 6], we propose a different type of nonlinear integral equation (NLIE) whose solution provides access to the effective central charge of both unitary and nonunitary minimal models. The equations can be found in section 2 , and are tested in section 3 . In 4 and 5 we extract some exact results and make a comparison with ultraviolet and infrared perturbation theory. The connection between the massive models and the Gross-Neveu models and other comments can be found in section 6 .

2. Our starting point is a set of nonlinear integral equations that encode the groundstate energy of the imaginary-coupled simply laced affine Toda field theories. Our interest in these theories lies in the fact that for values of the Toda coupling constant $\beta^{2}=p /(p+1)$ the theory can be consistently restricted to the massive $\Phi_{\text {adg }}$ perturbation of the unitary $W \mathcal{G}_{N}^{p, p+1}$ minimal models [17 22]. Moreover, the massive $\phi_{13}$ perturbation of the nonunitary $W A_{1}^{p, q}$ minimal models can be obtained from the sine-Gordon model by tuning the coupling to $\beta^{2}=p / q$. A similar result may be true for the nonunitary minimal models based on the other 


$$
\begin{array}{l|l}
A_{n-1} & M_{a}=M \sin (\pi a / h) / \sin (\pi / h) \quad 1 \leq a \leq n-1 \\
D_{n} & M_{n-1}=M_{n}=M / 2 \sin (\pi / h) \quad, \quad M_{a}=M \sin (\pi a / h) / \sin (\pi / h) \quad 1 \leq a \leq n-2 \\
E_{6} & M_{1}=M_{2}=M, M_{3}=2 M \cos (\pi / 4) \quad, \quad M_{4}=M_{5}=2 M \cos (\pi / 12) \\
& M_{6}=4 M \cos (\pi / 12) \cos (\pi / 4) \\
E_{7} & M_{1}=M, M_{2}=2 M \cos (5 \pi / 18) \quad, \quad M_{3}=2 M \cos (\pi / 9), M_{4}=2 M \cos (\pi / 18) \\
& M_{5}=2 M_{2} \cos (\pi / 18), M_{6}=2 M_{3} \cos (2 \pi / 9), M_{7}=2 M_{3} \cos (\pi / 18) \\
E_{8} & M_{1}=M, M_{2}=2 M \cos (\pi / 5), M_{3}=2 M \cos (\pi / 30), M_{4}=2 M_{2} \cos (7 \pi / 30) \\
& M_{5}=2 M_{2} \cos (2 \pi / 15), M_{6}=2 M_{2} \cos (\pi / 30) \\
& M_{7}=4 M_{2} \cos (\pi / 5) \cos (7 \pi / 30), M_{8}=4 M_{2} \cos (\pi / 5) \cos (2 \pi / 15)
\end{array}
$$

Table 1: The mass normalisation.

simply laced Lie algebras [23], and at the level of the NLIEs we do find the choice $\beta^{2}=p / q$ yields both unitary and nonunitary perturbed models.

The NLIEs describing the groundstate energy of the massive imaginary-coupled Toda field theories were first obtained in [24, 25], and have appeared in a different context in [26]. The effective central charge is defined in terms of $N$ functions which satisfy a set of coupled equations

$$
f^{(a)}(\theta)=-\frac{i}{2} m_{a} r e^{\theta}+i \pi \sum_{b=1} C_{a b}^{-1} \alpha_{b}+2 i \sum_{b=1}^{N}\left[\int_{\mathcal{C}} d \theta^{\prime} \varphi_{a b}\left(\theta-\theta^{\prime}\right) \Im m \ln \left(1+e^{f^{(b)}\left(\theta^{\prime}\right)}\right)\right] .
$$

The integration contour $\mathcal{C}$ runs just below the real axis while $r$ is built from the lightest mass $M$ of the theory and the cylinder size $R$ via $r=M R$. We have set $m_{a}=M_{a} / M$, where each mass $M_{a}$ is associated to a node of the Dynkin diagram via the labelling of [27, and is such that $\left(M_{1}, M_{2}, \ldots, M_{N}\right)$ forms an eigenvector of the Cartan matrix with eigenvalue $4 \sin ^{2}(\pi / 2 h)$. Our particular normalisation is given in table 1 . The kernel functions

$$
\varphi_{a b}(\theta)=\int_{-\infty}^{\infty} \frac{d k}{2 \pi} e^{i k \theta}\left(\delta_{a b}-\frac{\left.\sinh \left(\frac{\pi}{h}(\xi+1) k\right)\right)}{\sinh \left(\frac{\pi}{h} \xi k\right) \cosh \left(\frac{\pi}{h} k\right)} C_{a b}^{-1}(k)\right)
$$

are written in terms of the 'deformed' Cartan matrix $C_{a b}(k)$, which is equal to 2 if $a=b$, and $-1 / \cosh (\pi k / h)$ if nodes $a$ and $b$ of the relevant Dynkin diagram are connected. Note that at $k=0$ it reduces to the standard Cartan matrix $C_{a b}$. The exact effective central charge may be determined using

$$
c_{\mathrm{eff}}(r)=-\frac{6 r}{\pi^{2}} \sum_{a=1}^{N} m_{a}\left[\int_{\mathcal{C}} d \theta \sinh \theta \Im m \ln \left(1+e^{f^{(a)}(\theta)}\right)\right] .
$$

Due to the nonlinear nature of (9) and (11), $c_{\text {eff }}(r)$ is usually obtained by solving the equations numerically. However, like the TBA equations, the NLIEs can be exactly evaluated at the ultraviolet point [28], the result for the above equations being [25]

$$
c_{\mathrm{eff}}(0)=N-\frac{3 \xi}{\xi+1} \sum_{a, b=1}^{N} C_{a b}^{-1} \alpha_{a} \alpha_{b} .
$$


The effective central charge of the massive $\Phi_{\text {adj }}$ perturbation of $W \mathcal{G}_{N}^{p, q}$ is obtained by setting the parameter $\xi$ and the twists $\alpha=\left(\alpha_{1}, \alpha_{2}, \ldots, \alpha_{N}\right)$ to

$$
\xi=p /(q-p) \quad, \quad \alpha=(2 / p, 2 / p, \ldots, 2 / p) .
$$

The affine Toda coupling constant $\beta^{2}$ is related to $\xi$ via $\beta^{2}=\xi /(\xi+1)$, and the above ensures $\beta^{2}=p / q$. The choice of $\alpha$ is motivated by [29,30] for the $A_{1}$ related models, and the prescription given in 25] which yields the central charge of the $W \mathcal{G}$ minimal models rather than $c_{\text {eff }}(r)$. As a first check we insert (13) into (12), simplify using $12 \sum_{a, b=1}^{N} C_{a b}^{-1}=N h(h+1)$, and recover the expected UV effective central charge (3).

In accordance with (8) we modify the massive equations to interpolate from a model with $\xi=p /(q-p)$ to one with $\xi^{\prime}=(2 p-q) /(p-q)$, that is $\xi^{\prime}=\xi-1$. Motivated by [1],6], we associate two functions $f_{R}^{(a)}$ and $f_{L}^{(a)}$ to each node of the Dynkin diagram, introduce new kernels and twists such that the functions satisfy

$$
\begin{aligned}
& f_{R}^{(m)}(\theta)=-\frac{i}{2} m_{a} r e^{\theta}+i \pi \sum_{b=1}^{N} C_{a b}^{-1} \alpha_{b}^{\prime} \\
& +2 i \sum_{b=1}^{N}\left[\int_{\mathcal{C}} d \theta^{\prime} \phi_{a b}\left(\theta-\theta^{\prime}\right) \Im m \ln \left(1+e^{f_{R}^{(b)}\left(\theta^{\prime}\right)}\right)+\int_{\mathcal{C}} d \theta^{\prime} \chi_{a b}\left(\theta-\theta^{\prime}\right) \Im m \ln \left(1+e^{-f_{L}^{(b)}\left(\theta^{\prime}\right)}\right)\right], \\
& f_{L}^{(m)}(\theta)=-\frac{i}{2} m_{a} r e^{-\theta}-i \pi \sum_{b=1}^{N} C_{a b}^{-1} \alpha_{b}^{\prime} \\
& -2 i \sum_{b=1}^{N}\left[\int_{\mathcal{C}} d \theta^{\prime} \phi_{a b}\left(\theta-\theta^{\prime}\right) \Im m \ln \left(1+e^{-f_{L}^{(b)}\left(\theta^{\prime}\right)}\right)+\int_{\mathcal{C}} d \theta^{\prime} \chi_{a b}\left(\theta-\theta^{\prime}\right) \Im m \ln \left(1+e^{f_{R}^{(b)}\left(\theta^{\prime}\right)}\right)\right] .
\end{aligned}
$$

and replace the formula for the effective central charge with

$$
c_{\text {eff }}(r)=-\frac{6 r}{\pi^{2}} \sum_{a=1}^{N} m_{a}\left[\int_{\mathcal{C}} d \theta e^{\theta} \Im m \ln \left(1+e^{f_{R}^{(a)}(\theta)}\right)-\int_{\mathcal{C}} d \theta e^{-\theta} \Im m \ln \left(1+e^{-f_{L}^{(a)}(\theta)}\right)\right] .
$$

As explained in [1], we fix the kernel functions by considering the equations in the limits in which $r \rightarrow 0$ and $r \rightarrow \infty$. In the far infrared, the massless equations coincide with the ultraviolet limit of the massive equations, with kernel $\varphi_{a b}(\theta)$ replaced by $\phi_{a b}(\theta)$. Since this should describe a model with parameter $\xi-1$ we set $\phi_{a b}(\theta)$ to

$$
\phi_{a b}(\theta)=\int_{-\infty}^{\infty} \frac{d k}{2 \pi} e^{i k \theta}\left(\delta_{m t}-\frac{\sinh \left(\frac{\pi \xi}{h} k\right)}{\sinh \left(\frac{\pi}{h}(\xi-1) k\right) \cosh \left(\frac{\pi}{h} k\right)} C_{m t}^{-1}(k)\right) .
$$

For very small $r$, the massless equations should instead coincide with the ultraviolet limit of the massive equations with parameter $\xi$. After some manipulations [1], the fourier transformed massive and massless equations can be directly compared. With the tilde denoting the fourier transformed functions, the equations will match provided $\widetilde{\chi}_{a b}(k)$ and $\alpha_{a}^{\prime}$ satisfy

$$
\begin{aligned}
\widetilde{\varphi}_{a b}(k) & =\widetilde{\phi}_{a b}(k)+\sum_{c, d=1}^{N} \tilde{\chi}_{a c}(k)[\mathbb{1}-\widetilde{\phi}(k)]_{c d}^{-1} \tilde{\chi}_{d b}(k), \\
\alpha_{a} & =\alpha_{a}^{\prime}+\sum_{c, d=1}^{N} \tilde{\chi}_{a c}(0)[\mathbb{1}-\widetilde{\phi}(0)]_{c d}^{-1} \tilde{\chi}_{d b}(0) .
\end{aligned}
$$


Inserting the expressions for $\widetilde{\varphi}_{a b}$ and $\widetilde{\phi}_{a b}$ into $(18)$ we find

$$
\left(\frac{\sinh \left(\frac{\pi}{h} k\right)}{\sinh \left(\frac{\pi}{h}(\xi-1) k\right) \cosh \left(\frac{\pi}{h} k\right)}\right)^{2} C_{a b}^{-1}(k)=\sum_{c, d=1}^{N} \tilde{\chi}_{a c}(k) C_{c d}(k) \tilde{\chi}_{d b}(k) .
$$

After multiplying both sides by $C_{f a}(k)$ and summing over the index $a$, we can write the right hand side as a square: $(\widetilde{\chi}(k) C(k))_{f b}^{2}$. Taking the square root and inverting the fourier transform yields

$$
\chi_{a b}(\theta)= \pm \int \frac{d k}{2 \pi} e^{i k \theta} \frac{\sinh \left(\frac{\pi}{h} k\right)}{\sinh \left(\frac{\pi}{h}(\xi-1) k\right) \cosh \left(\frac{\pi}{h} k\right)} C_{a b}^{-1}(k) .
$$

The above only fixes $\chi_{a b}(\theta)$ up to a sign, but we find that choosing the negative sign results in an effective central charge consistent with a $W \mathcal{G}$ minimal model, whereas the other choice does not yield a recognisable formula for $c_{\text {eff }}(0)$. With the negative sign we find the new twists should be

$$
\alpha_{a}^{\prime}=\frac{\xi}{\xi-1} \alpha_{a}
$$

To avoid the pole in (22) at $\xi=1$, and the poles in the kernels which cross the real axis as $\xi$ falls below one, we only consider models with $2 p>q$ and therefore $\xi>1$. This is a sensible restriction since a flow of the form (8) with $2 p<q$ would have an infrared CFT labelled by $(2 p-q, p)$, the first of which is negative.

3. The massless NLIEs have ultraviolet and infrared values of $c_{\text {eff }}(r)$ which exactly match those of the conjectured flow (8) provided we continue to use the massive prescription for $\xi$ and $\alpha$ (13). We find

$$
c_{\mathrm{eff}}(0)=N\left(1-\frac{h(h+1)}{p q}\right) \quad, \quad c_{\mathrm{eff}}(\infty)=N\left(1-\frac{h(h+1)}{(2 p-q) p}\right) .
$$

The massless flows naturally fall into families indexed by an integer $J=q-p$ :

$$
W \mathcal{G}_{N}^{p, p+J}+\Phi_{\text {adj }} \rightarrow W \mathcal{G}_{N}^{p-J, p}
$$

At $J=1$ there is a unique family corresponding to the flows between the unitary minimal models. For these models we tested the massless NLIEs against the the TBA equations [10, 11], typically finding very good agreement. As expected the effective central charge was consistently monotonic. For $J>1$ there are $\varphi(J)$ different families, each of which interpolates between nonunitary models (here $\varphi$ denotes the Euler- $\varphi$ function). Solving the NLIEs for the nonunitary models, we found that $c_{\text {eff }}(r)$ increases away from its UV value, undergoes a number of oscillations and then settles down to the predicted IR value. The nonmonotonic behaviour of two families of flows is illustrated in Figures 1 and 2 .

4. To strengthen the validity of the conjectured equations we extract a number of further predictions, which are then compared with results from ultraviolet and infrared conformal perturbation theory.

The UV groundstate energy of a CFT perturbed by a primary field $\Phi$ of conformal dimension $\Delta_{\mathrm{UV}}$ is predicted to behave as [31, 32]

$$
E_{0}(R)=R B(\lambda)-\frac{\pi c_{\text {pert }}(r)}{6 R} \quad, \quad c_{\text {pert }}(r)=c_{\text {eff }}(0)+\sum_{j=1}^{\infty} C_{j}\left(\lambda R^{y}\right)^{j},
$$




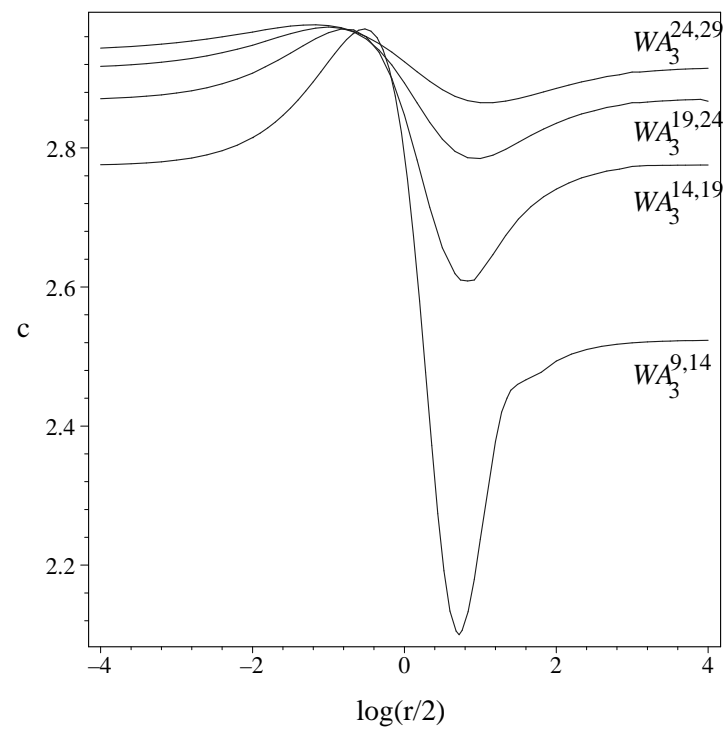

Figure 1: A family based on $W A_{3}$ with $J=6$.

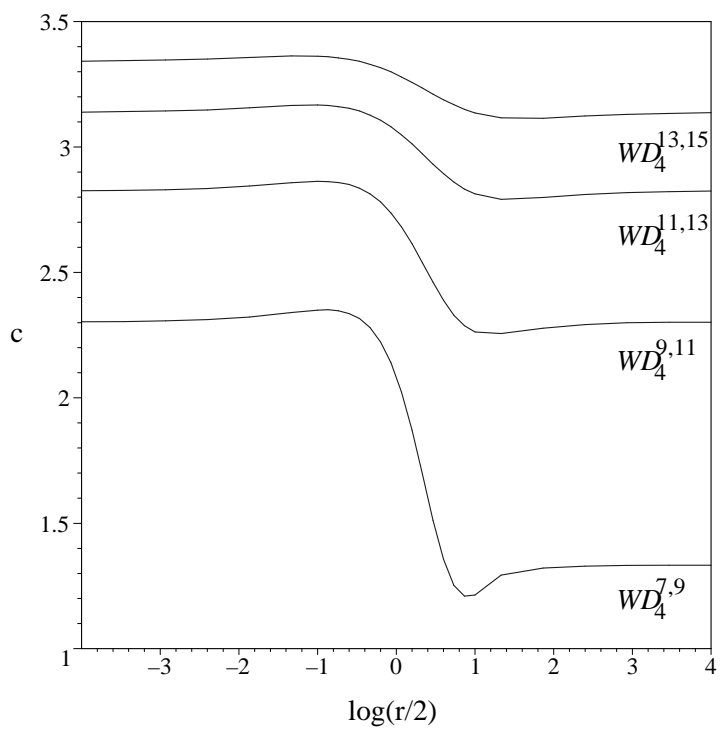

Figure 2: A family based on $W D_{4}$ with $J=2$.

where $y=2\left(1-\Delta_{\mathrm{UV}}\right)$ and the coefficients $C_{j}$ are proportional to the connected correlation functions of the perturbing field on the plane. The action (6) implies $\lambda$ and $M$ must satisfy

$$
\lambda=\kappa M^{y}
$$

for a dimensionless constant $\kappa$, further implying that $c_{\text {pert }}$ expands in powers of $r^{y}$. On the other hand, the $2(\xi+1) i \pi / h$ periodicity fof the nonlinear integral equations suggests that $c_{\text {eff }}(r)$ expands as a series in $r^{2 h /(1+\xi)}$, which will agree with $c_{\text {pert }}(r)$ provided $2\left(1-\Delta_{\mathrm{UV}}\right)=$ $2 h /(1+\xi)$. Substituting $p /(q-p)$ for $\xi$, we find the NLIEs predict a value of $\Delta_{\mathrm{UV}}$ which exactly matches the conformal dimension of $\Phi_{\text {adj }}(5)$.

The expansion

$$
c_{\mathrm{eff}}(r)=c_{\mathrm{eff}}(0)+B(r)+\sum_{j=1}^{\infty} c_{j}\left(r^{y}\right)^{j},
$$

differs from $c_{\text {pert }}(r)$ by the bulk term $B(r)$, but it may easily be extracted from the NLIEs. For this we need the leading asymptotics of the kernels as $\theta \rightarrow-\infty$. The denominator of the inverse deformed Cartan matrix has either a term of the form $\sinh (\pi k)\left(A_{n}\right)$ or $\cosh (\pi k / 2)$ $\left(D_{n}, E_{6}, E_{7}, E_{8}\right)$, both possibilities leading to a simple pole in the kernels $\phi_{a b}(\theta)$ and $\chi_{a b}(\theta)$ at $k=-i$. Moreover, the kernels $(17,21)$ have a pole at $k=-i h /(\xi-1)$, which contributes a term proportional to $e^{h \theta /(\xi-1)}$. Therefore we have

$$
\begin{aligned}
\phi_{a b}(\theta) & \sim \phi_{a b}^{(1)} e^{\theta}+p_{a b}^{(1)} e^{h \theta /(\xi-1)}+\ldots, \quad \theta \rightarrow-\infty, \\
\chi_{a b}(\theta) & \sim \chi_{a b}^{(1)} e^{\theta}+c_{a b}^{(1)} e^{h \theta /(\xi-1)}+\ldots, \quad \theta \rightarrow-\infty .
\end{aligned}
$$

${ }^{*}$ While the periodicity is easily extracted from the associated Bethe ansatz equations [25, 26, it is less trivial to see directly from the NLIEs. 
The expansion coefficients can be calculated by taking the appropriate residues, but we will only need $\phi_{1 a}^{(1)}$ and $\chi_{1 a}^{(1)}$, both of which can be written as

$$
\phi_{1 a}^{(1)}=\frac{\sin \left(\frac{\pi}{h} \xi\right) \sin \left(\frac{\pi}{h}\right)}{\pi \sin \left(\frac{\pi}{h}(\xi-1)\right) \nu(\mathcal{G})} m_{a} \quad, \quad \chi_{1 a}^{(1)}=\frac{\sin ^{2}\left(\frac{\pi}{h}\right)}{\pi \sin \left(\frac{\pi}{h}(\xi-1)\right) \nu(\mathcal{G})} m_{a}
$$

where

$$
\begin{array}{ll}
\nu\left(A_{n-1}\right)=1 \quad, \quad \nu\left(D_{n}\right)=1 / 2 \quad, \quad \nu\left(E_{6}\right)=\sqrt{2 / 3} \sin (\pi / 12) \\
\nu\left(E_{7}\right)=\frac{2}{\sqrt{3}} \sin (\pi / 18) \sin (2 \pi / 9) \quad, \quad \nu\left(E_{8}\right)=\frac{\sin (\pi / 30)}{2 \sqrt{3} \sin (\pi / 5)} .
\end{array}
$$

A small generalisation of the argument described in [1] yields the massive and massless bulk terms:

$$
B_{\text {massive }}(r)=-\frac{3 \sin \left(\frac{\pi}{h} \xi\right) \nu(\mathcal{G})}{2 \pi \sin \left(\frac{\pi}{h}(1+\xi)\right) \sin \left(\frac{\pi}{h}\right)} r^{2} \quad, \quad B_{\text {massless }}(r)=\frac{3 \nu(\mathcal{G})}{\left.2 \pi \sin \left(\frac{\pi}{h}(1+\xi)\right)\right)} r^{2} .
$$

The bulk term corresponding to the massive perturbation of the unitary minimal models has been calculated by Fateev [33] in the context of the associated coset. By analytically continuing the coset parameter $k$ to rational values (set $k=\xi-h$ ), we find Fateev's massive bulk term exactly coincides with ours for both unitary and nonunitary models.

The massless bulk terms are new, but we can make at least one concrete check before turning to numerics: the models with $p=h+1$ and $q=h+2$ correspond to the coset $\mathcal{G}_{N}^{1} \times$ $\mathcal{G}_{N}^{1} / \mathcal{G}_{N}^{2}$, for which the massive and massless perturbations are known to coincide [9, 34], as fortunately do our bulk terms at $\xi=h+1$.

The bulk terms have a simple pole whenever $\xi+1=m h$ for some integer $m$, which should cancel against one of the terms in the infinite sum so that the expansion (27) continues to be regular 35 37. In all cases the result, found by evaluating

$$
\lim _{\xi=m h-1} \frac{B(r)}{r^{2}}\left[r^{2}-r^{2 m \frac{h}{(\xi+1)}}\right],
$$

is a logarithmic term:

$$
\left.B_{\text {massive }}(r)\right|_{\xi=m h-1}=\frac{3 \nu(\mathcal{G})}{\pi^{2} m} r^{2} \ln r \quad,\left.\quad B_{\text {massless }}(r)\right|_{\xi=m h-1}=\frac{3(-1)^{m} \nu(\mathcal{G})}{\pi^{2} m} r^{2} \ln r .
$$

Now we are in position to compare (25) with (27), apart from one remaining difficulty. The perturbative coefficients $C_{j}$ are usually hard to calculate, and instead it is easier to estimate, from the massless and massive NLIEs respectively, the expansion coefficients $c_{j}$ and $\widetilde{c}_{j}$. The perturbative coefficients $C_{j}$ do not depend on $\lambda$, and if we assume the mass and crossover scales $M$ are equal we should find

$$
c_{j}=(-1)^{j} \widetilde{c}_{j}
$$

We include a small sample of our numerics in table 2. Such good agreement provides an excellent check on both the massive and massless NLIEs, the associated bulk terms and the above assumption on $M$.

5. Close to the infrared fixed point the model is described by the action of the infrared CFT plus an infinite number of contributions from irrelevant operators, resulting in a theory which 


\begin{tabular}{c|c|l}
\multicolumn{3}{c}{$W A_{2}^{7,10}+\Phi_{\mathrm{adj}}$} \\
$j$ & $\widetilde{c}_{j}$ & $c_{j}$ \\
\hline 0 & 1.657142856 & 1.657142856 \\
1 & -1.49526585 & 1.49526587 \\
2 & 0.01009599 & 0.01009595 \\
3 & -0.0015271 & 0.0015278 \\
4 & 0.0000603 & 0.0000602
\end{tabular}

\begin{tabular}{l|l|l}
\multicolumn{3}{|c}{$W D_{4}^{15,17}+\Phi_{\text {adj }}$} \\
$j$ & $\widetilde{c}_{j}$ & $c_{j}$ \\
\hline 0 & 3.34117647 & 3.34117646 \\
1 & -0.1962515 & 0.1962514 \\
2 & 0.067572 & 0.067573 \\
3 & 0.003602 & -0.003603 \\
4 & 0.00005 & 0.000008
\end{tabular}

Table 2: Comparison of the massive and massless UV coefficients, found via the NLIEs.

is unrenormalisable. However by considering the contribution of a finite number of fields it is still possible to make a comparison with results from either NLIEs such as ours or from TBA equations 38, 39. We consider

$$
\mathcal{A}=\mathcal{A}_{\mathrm{IR}}+g \int \psi d^{2} x+t \int T \bar{T} d^{2} x+\ldots
$$

where the (possibly missing) irrelevant field $\psi$ of dimension $\Delta_{\mathrm{IR}}$ and $T \bar{T}$ of dimension 2 belong to the infrared CFT. The couplings are related to the crossover scale $M$ as

$$
g=\kappa_{g} M^{2-2 \Delta_{I R}} \quad, \quad t=\kappa_{t} M^{-2} .
$$

The action implies $c_{\text {eff }}(r)$ has IR expansion

$$
c_{\mathrm{eff}}(r) \sim c_{\mathrm{eff}}(\infty)+\sum_{j=1}^{\infty} g_{j}\left(\kappa_{g} r\right)^{\left(2-2 \Delta_{\mathrm{IR}}\right) j}+\sum_{j=1}^{\infty} t_{j}\left(\kappa_{t} r\right)^{-2 j}+\ldots .
$$

From the NLIE point of view [1] corrections to $c_{\mathrm{eff}}(r)$ come from the $\theta \rightarrow-\infty$ asymptotic of $\chi_{a b}(\theta)$ given by (29), the $e^{h \theta /(\xi-1)}$ term generating a a series of the form $r^{-2 h /(\xi-1)}$. Comparing with the CPT expansion (38) leads to the prediction $\Delta_{\mathrm{IR}}=1+h /(\xi-1)$, which can be identified with the conformal dimension of the primary field $\Phi_{\text {adj }}$ with weights $\Omega=\Omega_{\text {id }}, \Omega^{\prime}=$ $\Omega_{\text {adj }}$.

We can also extract a prediction for $\kappa_{t}$ from the NLIEs. We start with the first two coefficients of the series generated by $T \bar{T}$ [39:

$$
t_{1}=-\frac{\pi^{3} c_{\mathrm{eff}}(\infty)^{2}}{6} \quad, \quad t_{2}=\frac{\pi^{6} c_{\mathrm{eff}}(\infty)^{3}}{18},
$$

and compare them with the coefficients of $r^{-2}$ and $r^{-4}$ found from the NLIEs. Adapting the TBA argument [37], we use the $e^{\theta}$ term in the expansion of $\chi_{a b}(\theta)$ to find

$$
c_{\mathrm{eff}}(r) \sim c_{\mathrm{eff}}(\infty)-\frac{2 \pi^{2}}{3} c_{\mathrm{eff}}^{2}(\infty) \chi_{11}^{(1)} r^{-2}+2 c_{\mathrm{eff}}^{3}(\infty)\left(\frac{2 \pi^{2}}{3} \chi_{11}^{(1)}\right)^{2} r^{-4}+\ldots
$$

The explicit form

$$
\chi_{11}^{(1)} r^{-2}=\frac{\sin ^{2}\left(\frac{\pi}{h}\right)}{\pi \sin \left(\frac{\pi}{h}(\xi-1)\right) \nu(\mathcal{G})} r^{-2}
$$


indicates a pole whenever $\xi-1=m^{\prime} h$ for some integer $m^{\prime}$. Evaluating as for the UV case we find (41) becomes

$$
\left.\chi_{11}^{(1)} r^{-2}\right|_{\xi=m^{\prime} h+1}=-\frac{2(-1)^{m^{\prime}} \sin ^{2}\left(\frac{\pi}{h}\right)}{\pi^{2} m^{\prime} \nu(\mathcal{G})} r^{-2} \ln r .
$$

The infrared expansion coefficients have been obtained numerically for the models $W A_{1}^{p, p+1}+$ $\phi_{13}, p=5, \ldots, 10$ in [37, 40], and they show good agreement with our predictions, while for $W A_{1}^{p, p+1}$ and $W D_{n+1}^{2 n+2,2 n+3}$ we find agreement with the theoretical results of 37. Finally, the effective central charge of the $\phi_{12}, \phi_{21}$ and $\phi_{15}$ perturbations of the Virasoro minimal models is exactly half that of certain $W A_{2}$ models:

$$
\begin{aligned}
W A_{1}^{p, q}+\phi_{12} & \leftrightarrow W A_{2}^{p, 2 q}+\Phi_{\mathrm{adj}} \\
W A_{1}^{p, q}+\phi_{21} & \leftrightarrow W A_{2}^{q, 2 p}+\Phi_{\mathrm{adj}} \\
W A_{1}^{p, q}+\phi_{15} & \leftrightarrow W A_{2}^{2 p, q}+\Phi_{\mathrm{adj}}
\end{aligned}
$$

Only the $\phi_{21}$ and $\phi_{15}$ perturbed models have a massless flow, and the IR expansion coefficients found in [1] match with those predicted above for the associated $W A_{2}$ model. The correspondence actually works for any value of $\xi$ since the $\phi_{12} / \phi_{21} / \phi_{15}$ NLIE is based on the tadpole diagram $T_{1}$, which is related by folding to $A_{2}$.

Finally, comparing (40, 41) to (38, 39) yields the promised prediction for $\kappa_{t}$ :

$$
\kappa_{t}=\frac{4 \sin ^{2}\left(\frac{\pi}{h}\right)}{\pi^{2} \sin \left(\frac{\pi}{h}(\xi-1)\right) \nu(\mathcal{G})} .
$$

6. We have shown that the function $c_{\text {eff }}(r)$ for nonunitary models interpolates from a CFT in the ultraviolet to an infrared CFT that has smaller effective central charge, but in a nonmonotonic way. It is likely that there is a function, as yet unknown, which monotically interpolates between the nonunitary CFTs and satisfies a 'nonunitary $c$-theorem'.

We'd like to make two further comments concerning the massive nonlinear integral equations. First, level-rank (or KNS) duality [23, 41] relates two nonunitary minimal models

$$
W A_{n-1}^{n, p}=W A_{p-n-1}^{p-n, p} \quad, \quad n, p \text { coprime },
$$

which both have a field $\Phi_{\text {adj }}$ with conformal dimension $\Delta=\left(p n-p-n^{2}\right) / p$, resulting in entirely equivalent perturbed theories. It is not at all obvious that the NLIEs based on $A_{n-1}$ and those based on $A_{p-n-1}$ at the appropriate values of $\xi$ will produce the same value of $c_{\text {eff }}(r)$, but provided the normalisation of the lightest mass is such that the coupling $\lambda$ is the same for both models, our numerical studies confirm this. By equating the massive bulk terms we find

$$
\sin (\pi /(p-n)) M_{[n-1]}=\sin (\pi / n) M_{[p-n-1]} ;
$$

the same result can also be deduced from the relation $\lambda=\kappa M^{y}$ given for the unitary minimal models in [33]. We can rule out a massless flow from $W A_{n-1}^{n, p}$ via $\Phi_{\text {adj }}$ by considering the conjectured IR model $W A_{n-1}^{2 n-p, n}$, a sensible CFT if $2 n-p>h$. Since $h=n$ this would require $p<n$, contradicting the assumption $n<p$.

As mentioned above, the massive $\phi_{15}$ and $\phi_{12}$ perturbations of the the Virasoro models $W A_{1}^{2, p}$ (for odd $p$ greater than 4) are integrable, and the groundstate energy can be found 
using a single NLIE based on $A_{2}^{(2)}$ [42], with appropriate tuning of the free parameters. Thus by level-rank duality the models $W A_{p-3}^{p-2, p}$ have up to two extra massive perturbations in addition to $\Phi_{\text {adj }}$ whose groundstate energy can be described in terms of a nonlinear integral equation.

Second, the massive NLIEs encode the finite-size effects of a further set of models obtained by sending $\xi=k-h$ to infinity. In this limit the perturbed $\operatorname{coset} \mathcal{G}_{N}^{k} \times \mathcal{G}_{N}^{1} / \mathcal{G}_{N}^{k+1}+\Phi_{\text {adj }}$ becomes the $\mathcal{G}$ Gross Neveu model [22, 43], with groundstate energy described in terms of an infinite number $(k . N)$ of coupled TBA equations. The NLIEs offer a clear advantage over the TBA as the $N$ equations can still be solved numerically at any value of $r$. The kernels

$$
\varphi_{a b}(\theta)=\int_{-\infty}^{\infty} \frac{d k}{2 \pi} e^{i k \theta}\left(\delta_{a b}-\frac{e^{\pi|k| / h}}{\cosh \left(\frac{\pi}{h} k\right)} C_{a b}^{-1}(k)\right)
$$

form part of the prefactors of the associated S-matrices [44]. The perturbation is (almost) marginal and the ultraviolet expansion of the effective central charge no longer has a simple power series form (27). By studying the NLIEs (numerically and analytically) we hope to uncover the expected logarithmic corrections to $c_{\text {eff }}(r)$. If this approach is successful there are a number of two-dimensional sigma models which have an interpretation as a perturbed conformal field theory [44]. Since in all cases the groundstate energy is described by an infinite number of TBA equations, it would also be interesting to extend the current set of NLIEs to include such models.

Finally we remark that even though the groundstate energy of the perturbed nonunitary

models is apparently real, recent results based on the $A_{2}^{(2)}$ models indicate the massive finite size spectrum may in general be complex [45]. It remains an open question to study the excited states of the massless models, perhaps via the massless NLIEs, to see if a similar result holds.

Acknowledgments - I'm grateful to Pascal Baseilhac, Patrick Dorey, André LeClair, Pierre Mathieu and Roberto Tateo for useful conversations. I also thank the EPSRC for a Research Fellowship and IPAM, UCLA for their hospitality.

\section{References}

[1] P. Dorey, C. Dunning and R. Tateo, Nucl. Phys. B578 (2000) 699

[2] A.B. Zamolodchikov, JETP Lett. 43 (1986) 730

[3] D. Boyanovsky and R. Holman, Nucl. Phys. B332 (1991) 359

[4] P. Fendley and H. Saleur, Nucl. Phys. B388 (1992) 609

[5] P. Fendley, H. Saleur and Al.B. Zamolodchikov, Int. J. Mod. Phys. A8 (1993) 5717

[6] Al. B. Zamolodchikov, Phys. Lett. B335 (1994) 436

[7] A. Bilal and J.L. Gervais, Phys. Lett. B206 (1998) 412; Nucl. Phys. B314 (1989) 646

[8] S.L. Lukyanov and V.A. Fateev, Sov. Sci. Rev. A15.2 (1990) 1

[9] Al. B. Zamolodchikov, Nucl. Phys. Lett. B366 (1991) 122

[10] M.J. Martins, Phys. Lett. B277 (1992) 301 
[11] F. Ravanini, Phys. Lett. B282 (1992) 73

[12] C. Crnkovic, G.M. Sotkov, M. Stanishkov, Phys. Lett. B226 (1989) 297

[13] A.B. Zamolodchikov, Sov. J. Nucl. Phys. 46 (1987) 1090

[14] A.W.W. Ludwig and J.L. Cardy, Nucl. Phys. B285 (1987) 687

[15] M. Lässig, Phys. Lett. B278 (1992) 439

[16] C. Ahn, Phys. Lett. B294 (1992) 204

[17] F.A. Smirnov, Nucl. Phys. B337 (1990) 156

[18] A. LeClair, Phys. Lett. B230 (1989) 103

[19] N. Reshetikhin and F. Smirnov, Comm. Math. Phys. 131 (1990) 157

[20] T. Eguchi and S.-K. Yang, Phys. Lett. B235 (1990) 282

[21] D. Bernard and A. LeClair, Nucl. Phys. B340 (1990) 721

[22] H.J. De Vega and V.A. Fateev, Int. J. Mod. Phys. A6 (1991) 3221

[23] A. Kuniba, T. Nakanishi and J. Suzuki, Nucl. Phys. B356 (1991) 750

[24] A. Mariottini, Degree thesis (in Italian), Bologna March 1996

[25] P. Zinn-Justin, J. Phys. A31 (1998) 6747

[26] P. Dorey, C. Dunning and R. Tateo, J. Phys. A33 (2000) 8427, hep-th/008039

[27] H. W. Braden, E. Corrigan, P. E. Dorey and R. Sasaki, Nucl. Phys. B338 (1990) 689

[28] C. Destri and H. J. De Vega, Nucl. Phys. B438 (1995) 413, hep-th/9407117

[29] D. Fioravanti, A. Mariottini, E. Quattrini and F. Ravanini, Phys. Lett. B390 (1997) 243

[30] G. Feverati, F. Ravanini and G. Takacs, Nucl. Phys. B570 (2000) 615

[31] Al. B. Zamolodchikov, Nucl. Phys. B342 (1990) 695

[32] T.R. Klassen and E. Melzer, Nucl. Phys. B350 (1991) 635

[33] V. A. Fateev, Phys. Lett. B324 (1994) 45

[34] P. E. Dorey and F. Ravanini, Nucl. Phys. B406 (1993) 708

[35] D.A. Huse and M.E. Fisher, J. Phys. C15 (1982) L585

[36] J.L. Cardy and G. Mussardo, Nucl. Phys. B410 (1993) 451,hep-th/9306028

[37] P. Dorey, R. Tateo and K.E. Thompson, Nucl. Phys. B470 (1996) 317

[38] Al. B. Zamolodchikov, Nucl. Phys. B358 (1991) 524

[39] Al. B. Zamolodchikov, Nucl. Phys. B358 (1991) 497

[40] G. Feverati, E. Quattrini and F. Ravanini, Phys. Lett. B374 (1996) 64

[41] D. Altschuler, M. Bauer and H. Saleur, J. Phys. A23 (1990) L789

[42] P. Dorey and R. Tateo, Nucl. Phys. B571 (2000) 583 
[43] C. Ahn, D. Bernard and A. LeClair, Nucl. Phys. B346 (1990) 409

[44] P. Fendley, Phys. Rev. Lett. 83 (1999) 4468; JHEP 0105 (2001) 050

[45] G. Takács and G. Watts, Nucl. Phys. B547 (1999) 538; 'RSOS revisted', hep-th/0203073 